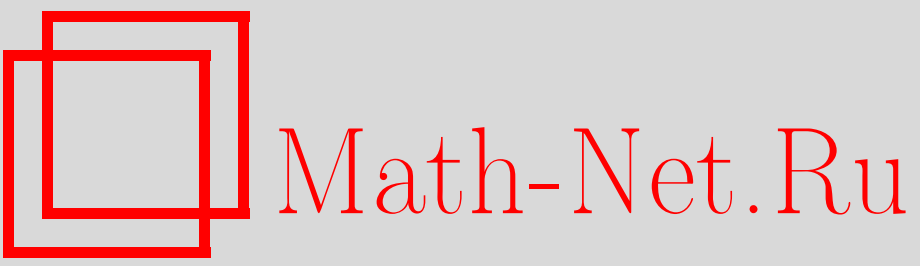

Ф. Калоджеро, Ж. П. Франсуаз, Нелинейные эволюционные ОДУ, имеющие много периодических решений, ТМФ, 2003, том 137, номер 3, 358-374

DOI: https://doi.org/10.4213/tmf278

Использование Общероссийского математического портала Math-Net.Ru подразумевает, что вы прочитали и согласны с пользовательским соглашением

http://www . mathnet.ru/rus/agreement

Параметры загрузки:

IP: 34.227 .88 .159

26 апреля 2023 г., 17:58:42 
ТЕОРЕТИЧЕСКАЯ

И МАТЕМАТИЧЕСКАЯ

ФИЗИКА

Том 137, № 3

декабрь, 2003

(C) 2003 г. $\quad$ Ф. Калоджеро*, Ж.-П. Франсуаз ${ }^{\dagger}$

\section{НЕЛИНЕЙНЫЕ ЭВОЛЮЦИОННЫЕ ОДУ, ИМЕЮШИЕ МНОГО ПЕРИОДИЧЕСКИХ РЕШЕНИЙ}

Некоторые (классы) одиночные автономные нелинейные эволюционные обыкновенные дифференциальные уравнения (ОДУ) сколь угодно высокого порядка идентифицируются как уравнения, которые могут быть модифицированы простым точным заданием условий, порождаюших однопараметрическое семейство деформированных автономных ОДУ со следующими свойствами: для всех положительных значений параметра деформации $\omega$ эти деформированные ОДУ имеют вполне периодические решения (c фиксированным периодом $\widetilde{T}=R \pi / \omega$, где $R$ - произвольное рациональное число), задаюшиеся (в контексте задачи Коши) открытыми областями начальных значений, причем мера этих областей в пространстве начальных значений зависит от параметра $\omega$, но, вообще говоря, является положительной (т.е. не обращается в нуль). Рассматриваются несколько примеров, включая однопараметрическую деформацию хорошо известного ОДУ третьего порядка, первоначально введенного Чези. Подробно обсуждается деформация уравнения Чези и строится точное открытое полуалгебраическое множество периодических орбит.

Ключевые слова: периодические решения, нелинейные осцилляторы, уравнение Чези.

\section{1. ВВЕДЕНИЕ И ОСНОВНЫЕ РЕЗУЛЬТАТЫ}

Недавно был найден подход [1], который позволил модифицировать некоторые (классы) автономные эволюционные уравнения так, чтобы после модификации они имели множество периодических решений, но не теряли своего автономного характера, т.е. демонстрировали явление, которое обусловлено свойством аналитичности по некоторой подходящим образом выбранной комплексной переменной типа "время", как это будет показано ниже. Этот подход применим как к одиночному нелинейному обыкновенному дифференциальному уравнению (ОДУ), так и к системе ОДУ, а также к дифференциальным уравнениям (системам уравнений) в частных производных [2]-[10]. Цель настоящей

*Dipartimento di Fisica, Università di Roma "La Sapienza", 00185 Roma; Instituto Nazionale di Fisica Nucleare, Sezione di Roma, Italy.

E-mail: francesco.calogero@roma1.infn.it, francesco.calogero@uniroma1.it

${ }^{\dagger}$ GSIB, 175 Rue du Chevaleret, Université Paris VI, 75013 Paris, France. E-mail: jpf@ccr.jussieu.fr 
работы - показать, что если рассматривается одиночное автономное нелинейное ОДу произвольного порядка $n$

$$
G\left(\zeta_{n}, \zeta_{n-1}, \zeta_{n-2}, \ldots, \zeta_{1}, \zeta\right)=0
$$

то этот подход применим при условии, что функция $G \equiv G\left(w_{n}, w_{n-1}, \ldots, w_{1}, w_{0}\right)$ обладает следующим $r$-сортирующим свойством:

$$
\begin{gathered}
G\left(\lambda^{n-r} w_{n}, \lambda^{n-1-r} w_{n-1}, \lambda^{n-2-r} w_{n-2}, \ldots, \lambda^{1-r} w_{1}, \lambda^{-r} w_{0}\right)= \\
=\lambda^{s} G\left(w_{n}, w_{n-1}, w_{n-2}, \ldots, w_{1}, w_{0}\right) .
\end{gathered}
$$

Здесь и ниже $\zeta \equiv \zeta(\tau)$ - (вообше говоря, комплексная) зависимая переменная, $\tau$ - (вообще говоря, комплексная) независимая переменная,

$$
\zeta_{m} \equiv \zeta_{m}(\tau) \equiv \frac{d^{m} \zeta(\tau)}{d \tau^{m}}, \quad m=0,1,2, \ldots
$$

$r$ - произвольное рациональное число, скажем

$$
r=\frac{p}{q}
$$

где $p, q$ - два произвольных взаимно простых целых числа (в дальнейшем для определенности предполагается, что $q$ - положительно, $q>0), s$ - произвольная постоянная (которая, вообше говоря, играет второстепенную роль), $\lambda$ - произвольный масштабирующий параметр, а функция $G \equiv G\left(w_{n}, w_{n-1}, w_{n-2}, \ldots, w_{1}, w_{0}\right)$ предполагается аналитически зависящей от все $x$ своих $n+1$ аргументов $w_{m}, m=0,1,2, \ldots, n$ (и такой, что уравнение (1.1) действительно квалифицируется как “уравнение эволюции”, а именно оно “разрешимо" относительно высшей производной $\left.\zeta_{n}\right)$.

Это автономное ОДУ (1.1) является немодифицированной версией, которая с помошью рассматриваемого "подхода" (см. ниже) преврашается в новое ("деформированное” ) автономное ОДУ для новой (вообше говоря, комплексной) зависимой переменной $z \equiv z(t)$. Затем предполагается, что эта функция зависит от действительной независимой переменной $t$ (от "времени") и связана с переменной $\zeta(\tau)$ простой заменой (зависимых и независимых) переменных, а именно с помощью подхода, описанного в разделе 2. Деформированное ОДУ получается из (1.1) заменой

$$
\zeta_{m} \Rightarrow z_{m}+\sum_{l=1}^{m}\left[a_{m l}(r)(i q \omega)^{l} z_{m-l}\right], \quad m=0,1,2, \ldots
$$

где, конечно, по аналогии с (1.1б) мы имеем

$$
z_{m} \equiv z_{m}(t) \equiv \frac{d^{m} z(t)}{d t^{m}}, \quad m=0,1,2, \ldots
$$


Постоянные $a_{m l}(r)$ в $(1.2 \mathrm{a})$ являются универсальными коэффициентами, которые легко оценить с помощью рекуррентных вычислений (см. ниже).

После замены (1.2) автономное ОДУ (1.1), которому удовлетворяет переменная $\zeta \equiv$ $\zeta(\tau)$, модифицируется ("деформируется") в автономное ОДУ, которому удовлетворяет переменная $z \equiv z(t)$. Характерной чертой этого уравнения является то, что оно имеет действительный параметр $\omega$ (см. (1.2а)) и обладает следующими свойствами: при $\omega=0$ новое уравнение не отличается от немодифицированного (с точностью до тривиальных изменений обозначений, см. (1.2)); в дальнейшем в случае $\omega \neq 0$ без ограничения общности мы будем предполагать, что параметр $\omega$ положительный, т.е.

$$
\omega>0 \text {. }
$$

В этом случае деформированное ОДУ, полученное из (1.1) с помошњю замены (1.2), имеет множество вполне периодических решений, более точно (в контексте задачи Коши), оно имеет вполне периодические решения, которые задаются открытым множеством начальных значений, и это множество, конечно, имеет ненулевую меру в пространстве начальных значений. Полная периодичность этих решений,

$$
z(t+\widetilde{T})=z(t)
$$

характеризуется периодом $\widetilde{T}$, который, вообше говоря, является целым числом, кратным основному периоду $T$,

$$
T=\frac{2 \pi q}{\omega},
$$

соответствуюшему параметру дефоормации $\omega$ и “знаменателю" $q$ (см. (1.1г)).

В частности, при $n=4$ (или меньшем значении $n$, если функция $G \equiv G\left(w_{4}, w_{3}, w_{2}\right.$, $\left.w_{1}, w_{0}\right)$ не зависит от своего первого аргумента (аргументов)) деформированное ОДУ, полученное из (1.1) с помощью (1.2), имеет вид

$$
\begin{aligned}
G(\dddot{z}+ & 2 i(2 p-3 q) \omega \dddot{z}+\left(6 p^{2}-18 p q+11 q^{2}\right) \omega^{2} \ddot{z}- \\
& -i\left(4 p^{3}-18 p^{2} q+21 p q^{2}-3 q^{3}\right) \omega^{4} \dot{z}+p(p-q)(p-2 q)(p-3 q) \omega^{4} z, \\
& \dddot{z}+3 i(p-q) \omega \ddot{z}-\left(3 p^{2}-6 p q+2 q^{2}\right) \omega^{2} \dot{z}-i p(p-q)(p-2 q) \omega^{3} z, \\
& \left.\ddot{z}+i(2 p-q) \omega \dot{z}-p(p-q) \omega^{2} z, \dot{z}+i p \omega z, z\right)=0 .
\end{aligned}
$$

Здесь и ниже точки над символом обозначают дифференцирование по (действительной) переменной $t$ (по “времени”) и два целых числа $p, q$, так же как и рациональное число $r$, определяются, как и раньше (см. (1.1г)).

В разделе 2 мы описываем наш подход (фактически заключающийся в замене переменных) и затем доказьваем основной результат настоящей статьи. Именно, мы доказываем, что уравнение эволюции, полученное с помощью (1.2) из (1.1) (включаюшее (1.5) как частный случай явного уравнения), имеет вполне периодические решения с периодом $\widetilde{T}$ (см. (1.3)), задаюшиеся (в контексте задачи Коши) открытой областью 
начальных значений, которая имеет ненулевую меру в пространстве таких начальных значений. Границы этой области, вообше говоря, вместо этих решений задают решения, которые становятся особыми за конечное время.

Также представляет интерес поведение решений, которые задаются (в контексте задачи Коши) точками, лежащими вне этой области. Для некоторых ОДУ типа (1.1) с заменой (1.2) (некоторых специальных видов рассматриваемых уравнений) все их несингулярные решения являются вполне периодическими, но, возможно, имеют разные периоды, которые, однако, все являются кратными основному периоду $T$ (см. (1.4)). Для других типов этих ОДУ имеются определенные области начальных значений, которые задают периодические решения (с такими периодами), но также сушествуют области, которые задают непериодические, возможно "хаотические", решения. Это обшее явление (включая "переход к хаосу") в поведении решений эволюционных ОДУ этого класса, т.е. (1.1) с заменой (1.2), в настоящее время хорошо понято, так как были изучены некоторые примеры [7]-[10]. Однако анализ этого явления для рассматриваемых здесь ОДУ лежит за пределами данной работы, так как для его проведения требуется рассмотрение каждого случая в отдельности. Ниже мы приводим (см. замечание 4 и конец раздела 2) конкретные решения, которыми обладает целый класс ОДУ, которые можно получить из (1.1) с помошью замены (1.2) и в поведении которых просматриваются эти явления. Более того, в разделе 3 , где в качестве примеров рассматриваются некоторые уравнения эволюции (ОДУ) из класса (1.1) (в частности, все уравнения полиномиального типа, порядок которых не выше четвертого), ограничения (в виде явных неравенств) на начальные значения, достаточные для того, чтобы гарантировать полную периодичность соответствующего решения, приводятся только в одном конкретном случае, а именно в случае деформированной версии хорошо известного ОДУ третьего порядка, первоначально введенного Чези [11]. В приложение мы выносим некоторые вычислительные аспекты, что позволит нам не прерьвать основное изложение.

Закончим введение некоторыми замечаниями.

ЗАмЕчаниЕ 1. Так как автономные ОДУ представляют наибольший (теоретический и прикладной) интерес, в этой статье основное внимание уделяется классу немодифицированных ОДУ, которые сами являются автономными и порождают деформированные ОДУ, которые также являются автономными. Но этот подход также применим, если немодифицированные или деформированные ОДУ, или и те и другие не являются автономными (см., например, [6]). В этом случае можно отказаться от $r$-сортирующего свойства (1.1б)).

ЗАмЕЧАнИЕ 2. Деформированное ОДУ, полученное из (1.1) с помошью (1.2), является, вообше говоря, комплексным (см. (1.2) и характерный пример (1.5)). Но, конечно, оно может быть преобразовано в систему двух действительных спаренных ОДУ для двух действительных зависимых переменных $x \equiv x(t), y \equiv y(t)$, если положить

$$
z \equiv z(t)=x+i y \equiv x(t)+i y(t)
$$


и затем рассматривать два ОДУ, которые получаются в результате приравнивания нулю действительной и мнимой частей ОДУ, полученного из (1.1) с помощью (1.2) с учетом (1.6). Другая возможность состоит в том, чтобы использовать фазово-амплитудное представление комплексного числа $z(t)=\rho(t) e^{i \theta(t)}$.

ЗАМЕЧАНИЕ 3 . Примерами ОДУ $n$-го порядка, которые обладают $r$-сортируюшим свойством (1.1б), являются уравнения

$$
\begin{gathered}
F\left(\zeta_{n} \zeta^{(n-r) / r}, \zeta_{n-1} \zeta^{[(n-1)-r] / r}, \zeta_{n-2} \zeta^{[(n-2)-r] / r}, \ldots, \zeta_{2} \zeta^{(2-r) / r}, \zeta_{1} \zeta^{(1-r) / r}\right)=0 \\
F\left(\zeta_{n} \zeta_{1}^{(n-r) /(r-1)}, \zeta_{n-1} \zeta_{1}^{[(n-1)-r] /(r-1)}, \ldots, \zeta_{2} \zeta_{1}^{(2-r) /(r-1)}, \zeta \zeta_{1}^{-r /(r-1)}\right)=0 \\
F\left(\zeta_{n} \zeta_{1}^{-n}, \zeta_{n-1} \zeta_{1}^{-(n-1)}, \zeta_{n-2} \zeta_{1}^{-(n-2)}, \ldots, \zeta_{2} \zeta_{1}^{-2}, \zeta\right)=0
\end{gathered}
$$

где от функций $F$ теперь требуется только, чтобы они аналитически зависели от всех своих $n$ аргументов, но при этом не удовлетворяли бы какому-либо сортирующему свойству. В действительности ясно, что эти ОДУ удовлетворяют $r$-сортирующему свойству (1.1б) при $s=0$. Заметим, что формула (1.9) - это частный случай формулы (1.8) при $r=0$ (соответствуюший случаю $p=0$ и, без ограничения обшности, случаю $q=1$, cM. $(1.1 \Gamma))$.

ЗАМЕчАНИЕ 4. Из r-сортирующего свойства (1.1б) следует (см. приложение), что ОДУ (1.1а) допускает решение(я) специального вида

$$
\zeta(\tau)=c\left(\tau-\tau_{b}\right)^{r},
$$

где $\tau_{b}$ - произвольная, вообше говоря комплексная, константа (нижний индекс $b$ служит напоминанием о том, что функция (1.10a), вообше говоря, имеет алгебраическую (см. $(1.1 г))$ точку ветвления при $\tau=\tau_{b}$ ), тогда как константа(ы) $c$ должна(ы) удовлетворять (вообше говоря, алгебраическому) уравнению

$$
G\left((r)_{n} c,(r)_{n-1} c, \ldots, r c, c\right)=0 .
$$

Здесь и ниже мы пользуемся стандартными обозначениями

$$
(x)_{0}=1, \quad(x)_{m}=x(x-1) \ldots(x-m+1), \quad m=1,2,3, \ldots .
$$

Кроме того, ясно, что если линеаризовать уравнение (1.1a) в окрестности решения (1.10), полагая

$$
\zeta(\tau)=c\left(\tau-\tau_{b}\right)^{r}+\varepsilon \varphi(\tau),
$$

где $\varepsilon-$ (малый) параметр разложения, то при $\varphi \equiv \varphi(\tau)$ получается (см. приложение) линейное ОДУ $n$-го порядка, все решения которого в обшем случае также являются простыми степенями (суммой простых степеней),

$$
\varphi(\tau)=\sum_{m=1}^{n} C_{m}\left(\tau-\tau_{b}\right)^{\alpha_{m}},
$$


где $n$ констант $C_{m}$, конечно, $n$ роизвольны и $n$ показателей $\alpha_{m}$ - это $n$ корней следующего полиномиального уравнения $n$-го порядка относительно $\alpha$ :

$$
\sum_{m=0}^{n} g_{m}(\alpha)_{m}=0,
$$

где коэффициенты $g_{m}$ - частные производные функции $G \equiv G\left(w_{n}, w_{n-1}, \ldots, w_{1}, w_{0}\right)$, вычисляемые для тех же значений $n+1$ аргументов $w_{n}, w_{n-1}, \ldots, w_{1}, w_{0}$, которые присутствуют в левой части уравнения (1.10б):

$$
g_{m}=\frac{\partial G}{\partial w_{m}}\left((r)_{n} c,(r)_{n-1} c, \ldots, r c, c\right)
$$

где константа $c,-$ конечно, та же самая константа, которая появляется в (1.11а) и удовлетворяет (1.10б).

ЗАмЕЧАнИЕ 5. Очевидно, что степень ОДУ $n$-го порядка (1.1а) можно, вообще говоря, понизить до $n-1$, принимая во внимание автономный характер этого уравнения, а именно полагая

$$
\zeta^{\prime} \equiv \zeta_{1}=u(\zeta)
$$

так как в соответствии с этим определением ОДУ (1.1) становится неавтономным ОДУ порядка $n-1$, имеющим зависимую переменную $u \equiv u(\zeta)$ и независимую переменную $\zeta$, и, конечно, любое решение $u \equiv u(\zeta)$ этого ОДУ, будучи подставлено в (1.12), дает решение в квадратурах уравнения (1.1).

Кроме того, дополнительное понижение на порядок, вообше говоря, возможно, если принять во внимание $r$-сортируюшее свойство (1.1б) и соответствуюшую симметрию ОДУ (1.1). Значит, в общем случае возможно свести к квадратурам решение ОДУ (1.1) не только при $n=1$ (когда это действительно с очевидностью следует из самого уравнения (1.1)), но и при $n=2$, и, конечно, это также верно для деформированных версий (см. (1.2)) этих ОДУ, получаемых, как будет подробно показано в разделе 2, просто заменой переменных ("наш подход"). Может показаться, что этот факт уменьшает значение главного результата данной статьи, касающегося свойства периодичности решений модифицированных уравнений, в применении к ОДУ порядка меньше третьего $(n=1,2)$, однако фактически, даже когда уравнения разрешимы в квадратурах, бывает довольно трудно проверить, обладают ли эти уравнения свойствами периодичности.

\section{2. ОПИСАНИЕ ПОДХОДА И ДОКАЗАТЕЛЬСТВО ОСНОВНОГО РЕЗУЛЬТАТА}

Введем новую независимую переменную $t$, которую мы будем всегда считать дейсmвительной ("время"), и новую комплексную зависимую переменную $z \equiv z(t)$, полагая

$$
\begin{aligned}
z(t) & =e^{-i p \omega t} \zeta(\tau), \\
\tau & =\frac{e^{i q \omega t}-1}{i q \omega} .
\end{aligned}
$$


Здесь мы пользуемся обозначениями, введенными в разделе 1: в частности, $\omega-n о л о-$ жительный параметр (см. (1.2в)), а $p, q$ - два произвольных взаимно простых целых числа $(q>0)$, связанных с рациональным параметром $r$ соотношением $(1.1 \Gamma))$.

Легко можно получить следуюшие соотношения:

$$
\begin{aligned}
\zeta^{\prime}= & e^{i(p-q) \omega t}[\dot{z}+i p \omega z], \\
\zeta^{\prime \prime}= & e^{i(p-2 q) \omega t}\left[\ddot{z}+i(2 p-q) \omega \dot{z}-p(p-q) \omega^{2} z\right], \\
\zeta^{\prime \prime \prime}= & e^{i(p-3 q) \omega t}[\dddot{z}+3 i(p-q) \omega \ddot{z}- \\
& \left.-\left(3 p^{2}-6 p q+2 q^{2}\right) \omega^{2} \dot{z}-i p(p-q)(p-2 q) \omega^{3} z\right] \\
\zeta^{\prime \prime \prime \prime}= & e^{i(p-4 q) \omega t}[\dddot{z}+2 i(2 p-3 q) \omega \dddot{z}+ \\
& +\left(6 p^{2}-18 p q+11 q^{2}\right) \omega^{2} \ddot{z}- \\
& -i\left(4 p^{3}-18 p^{2} q+21 p q^{2}-3 q^{3}\right) \omega^{4} \dot{z}+ \\
& \left.+p(p-q)(p-2 q)(p-3 q) \omega^{4} z\right],
\end{aligned}
$$

а также

$$
\zeta_{m}=e^{i(p-m q) \omega t}\left\{z_{m}+\sum_{l=1}^{m}\left[a_{m l}(r)(i q \omega)^{l} z_{m-l}\right]\right\}, \quad m=1,2,3, \ldots
$$

Здесь мы считаем, что обозначения очевидны: штрихи обозначают производные по $\tau$, точки - производные по $t$, а $\zeta_{m}$ и $z_{m}$ определены соответственно соотношениями (1.1в) и (1.2б). Коэффициенты $a_{m l}(r)$ можно легко оценить с помощью рекуррентных вычислений (см. приложение).

Из формул (2.2д), (2.1а) и (1.1б) следует, что с помощью замены переменных (2.1) из автономного ОДУ (1.1) можно легко получить автономное ОДУ для $z \equiv z(t)$, что получается из (1.1) с помощью замены (1.2). В частности, ясно, что ОДУ (1.5) получается из (1.1a) при $n=4$ с помошью замены (2.2б)-(2.2г) и (2.1a).

Теперь мы докажем основные свойства деформированного ОДУ, полученного с помощью замены переменных (2.1) из (1.1), а именно мы покажем, что это деформированное ОДУ имеет вполне периодические решения с периодом $\widetilde{T}$ (см. (1.3)), задаюшиеся (в контексте задачи Коши) некоторой открытой областью начальных значений, имеющей ненулевую меру в пространстве таких начальных значений.

Сначала заметим, что так как действительная переменная $t$ ("время") возрастает от своего начального значения, скажем $t=0$, до $t=\infty$, то соответствуюшая переменная $\tau$ продолжает вращаться против часовой стрелки вдоль окружности $\widetilde{C}$, диаметр которой лежит на верхней части мнимой оси комплексной $\tau$-плоскости, один его конец находится в точке $\tau=0$ (что соответствует значению $t=0(\bmod T))$, а другой конец - в точке $\tau=2 i /(q \omega)$ (что соответствует значению $t=T / 2(\bmod T))($ см. $(2.1 \mathrm{a})$ и $(1.4))$. Следовательно, если решение $\zeta(\tau)$ уравнения (1.1) является голоморфнылм (или просто мероморфнылм) внутри этой окружности $\widetilde{C}$ и не является сингулярным на этой окружности $\widetilde{C}$, то соответствуюшее решение $z(t)$ деформированного ОДУ, полученного из $(1.1)$ с 
помошью замены переменных (2.1) (а именно, с помошью замены (1.2)), очевидно, является вполне периодическим с периодом

$$
\widetilde{T}=M(p, q) T
$$

где $M(p, q)$ - наименьшее общее кратное чисел $p$ и $q($ см. (2.1), (1.3) и (1.4)).

С другой стороны, общая теорема существования, единственности и аналитичности решения задачи Коши для эволюционных ОДУ гарантирует, что решение $\zeta(\tau)$ уравнения (1.1) действительно является голоморфным в круговом диске $D$ с центром в начале координат $\tau=0$ комплексной $\tau$-плоскости, радиус которого $\tau_{D}$ зависит (для заданного автономного ОДУ, как в нашем случае) только от начальных значений $\zeta_{m}(0)$, $m=0,1, \ldots, n-1$, и, вообше говоря, может быть выбран сколь угодно большим, если потребовать, чтобы эти начальные значения удовлетворяли соответствующим неравенствам [12]. Однако на самом деле достаточно потребовать, чтобы величина $\tau_{D}$ была больше, чем диаметр окружности $\widetilde{C}$,

$$
\tau_{D}>\frac{2}{q \omega}
$$

для того чтобы решение $\zeta(\tau)$ уравнения (1.1) было голоморфным в (замкнутом) круговом диске $C$, лежашем внутри окружности $\widetilde{C}$; тогда соответствуюшее решение $z(t)$ деформированного уравнения, полученное из (1.1) с помошью (2.1) или, что эквивалентно, с помощью (1.2), будет вполне периодическим с периодом $\widetilde{T}$ (см. (1.4), (2.2г)).

Теперь для завершения доказательства основного результата достаточно заметить, что открытая область начальных значений $\zeta_{m}(0), m=0,1, \ldots, n-1$, которые обеспечивают выполнение (2.2д) и таким образом гарантируют полную периодичность решения $z(t)$, отображается на открытую область начальных значений $z_{m}(0), m=0,1, \ldots, n-1$, поскольку из (2.2д) следует

$$
\zeta_{m}(0)=z_{m}(0)+\sum_{l=1}^{m}\left[a_{m l}(r)(i q \omega)^{l} z_{m-l}(0)\right], \quad m=0,1,2, \ldots,
$$

или, что эквивалентно,

$$
z_{m}(0)=\zeta_{m}(0)+\sum_{l=1}^{m}\left[b_{m l}(r)(i q \omega)^{l} \zeta_{m-l}(0)\right], \quad m=0,1,2, \ldots
$$

где коэффициенты $b_{m l}(r)$ могут быть легко получены с помощью рекуррентных вычислений из коэффишиентов $a_{m l}(r)$.

Таким образом, ясно, что если решение $\zeta(\tau)$ немодифицированного эволюционного ОДУ (1.1) имеет сингулярность в точке $\tau=\tau_{s}$, которая в точности попадает внутрь окружности $\widetilde{C}$, то соответствуюшее решение $z(t)$ деформированных ОДУ, полученных 
из (1.1) с помощью (2.1) или, что эквивалентно, с помощью (1.2), становится сингулярным при конечном действительном значении $t=t_{s}$ таком, что

$$
\tau_{s}=\frac{e^{i q \omega t_{s}}-1}{i q \omega}
$$

так как очевидно, что условие попадания $\tau_{s}$ на $\widetilde{C}$ в точности соответствует (см. (2.1a)) требованию, чтобы $t_{s}$ было действительной величиной.

Завершим этот раздел следуюшим замечанием.

ЗАмечаниЕ 6. Соответствуюшее частному “решению подобия” (1.10) немодифицированного ОДУ (1.1) деформированное ОДУ, которое получается из (1.1) с помошью (2.1) или, что эквивалентно, с помошью (1.2), имеет частное решение

$$
\begin{aligned}
& z(t)=c e^{-i p \omega t}\left\{-\tau_{b}+\frac{e^{i q \omega t}-1}{i q \omega}\right\}^{r}, \\
& z(t)=c\left\{-\tau_{b} e^{-i q \omega t}+\frac{1-e^{-i q \omega t}}{i q \omega}\right\}^{r},
\end{aligned}
$$

где константа $c$, конечно, должна быть решением алгебраического или трансцендентного уравнения (1.10б), тогда как (вообше говоря, комплексная) константа $\tau_{b}$ произвольна. Так же легко проверяется, что решение (2.7) вполне периодическое с периодом $\widetilde{T}$ (см. (2.3)), если $\tau_{b}$ лежит вне окружности $\widetilde{C}$,

$$
\left|\tau_{b}-\frac{i}{q \omega}\right|>\frac{1}{q \omega}
$$

(что согласуется с проведенным выше анализом). Это решение может быть вполне периодическим с периодом, меньшим или равным $T$ (см. (1.4)), если, наоборот, $\tau_{b}$ попадает внутрь окружности $\widetilde{C}$,

$$
\left|\tau_{b}-\frac{i}{q \omega}\right|<\frac{1}{q \omega} .
$$

Наконец, это решение становится сингулярным за конечное время $t_{s}$, которое определяется соотношением $(2.6) \quad(\bmod T)$ при соответствуюшем выборе $\tau_{b}=\tau_{s}($ cм. $(2.6))$ таком, чтобы оно попадало в точности на окружность $\widetilde{C}$ в комплексной $\tau$-плоскости.

\section{3. ПРИМЕРЫ: ПОЛИНОМИАЛЬНЫЕ ОДУ}

В этом разделе мы кратко обсудим несколько примеров, включающих, в частности, класс ОДУ типа (1.1), которые линейны относительно старшей производной и полиномиальны относительно всех остальных,

$$
\zeta_{n}=\sum_{\underline{m}} c(\underline{m}) \prod_{l=0}^{n-1}\left[\zeta_{l}\right]^{m_{l}}
$$


где символ $\underline{m} \equiv\left(m_{0}, m_{1}, \ldots, m_{n-1}\right)$ обозначает вектор размерности $n$, компонентами которого являются $n$ неотрицательных иельх $x$ чисел $m_{l}$, стоящих в показателе степени, а сумма в правой части этого эволюционного ОДУ берется по всем множествам тех значений $n$ неотрицательных цельх показателей $m_{l}$, которые удовлетворяют условию

$$
p \sum_{l=0}^{n-1} m_{l}-q \sum_{l=1}^{n-1} l m_{l}=p-q n,
$$

или, что эквивалентно (см. (1.1г)),

$$
\sum_{l=0}^{n-1}(r-l) m_{l}=r-n
$$

Константы $c(\underline{m})$ в (3.1а) произвольные (возможно, комплексные), а также здесь и ниже мы будем использовать обозначения (1.1в), так же как и (1.1г), где $p$ и $q>0$ - два взаимно простых числа.

Легко можно проверить, что этот класс эволюционных ОДУ (3.1) принадлежит классу (1.1) (в частности, эти уравнения удовлетворяют $r$-сортируюшему условию $(1.16)$ с $s=n-r)$. Также ясно, что условие (3.1в), где

$$
r=\left(n-\sum_{l=1}^{n} l m_{l}\right) /\left(1-\sum_{l=0}^{n} m_{l}\right)
$$

всегда выполняется, если только один из коэффициентов $c(\underline{m})$, появляющихся в правой части уравнения (3.1a), не обрашается в нуль. А именно, ОДУ

$$
\zeta_{n}=c(\underline{m}) \prod_{l=0}^{n-1}\left[\zeta_{l}\right]^{m_{l}}
$$

при любом выборе неотрицательного целочисленного $n$-мерного вектора $\underline{m} \equiv\left(m_{0}\right.$, $\left.m_{1}, \ldots, m_{n-1}\right)$, принадлежит классу $(1.1)$, так как оно очевидным образом удовлетворяет $r$-сортируюшему условию (1.1б), где $s=n-r$ и рациональное число $r$ определяется соотношением (3.2). В списке полиномиальных ОДУ, приведенном ниже, мы систематически не приводим ОДУ этого простого типа.

Теперь перечислим эволюционные ОДУ типа (3.1) порядка $n=2,3,4$. Здесь и ниже коэффициенты $c(\underline{m})$ являются произвольными (возможно, комплексными) константами, а символом $f$ (возможно, несушим некоторый индекс, когда нужно различать несколько таких функций) мы обозначаем функцию, зависящую аналитически от всех своих аргументов, но в остальном произвольную. Это условие является достаточным для того, чтобы гарантировать, что после деформации (1.2) приведенное ниже ОДУ действительно имеет много периодических решений. Мы здесь не приводим дополнительные условия, которым должны удовлетворять коэффициенты $c(\underline{m})$ или функции $f$ в каждом 
отдельном случае, чтобы гарантировать полиномиальный характер правых частей приводимых ниже ОДУ, поскольку они получаются в каждом отдельном случае непосредственной проверкой (конечно, достаточным условием является то, что сами функции $f$ полиномиальны по всем своим аргументам; в некоторых случаях это условие также является необходимым.). Для каждого приведенного ниже ОДУ мы также выписьваем соответствующие условия на значения двух взаимно простых целых чисел $p$ и $q>0$, которые характеризуют (усердный читатель может легко это проверить с помощью (2.2)) преобразование (2.1), переводящее эти недеформированные уравнения в частных производных в соответствуюшие им деформированные уравнения (см. (1.2), а также более точные формулы, следующие из (2.2)). Выбор приведенных ниже ОДУ, как и порядок их расположения, в основном обусловлен "эстетическими” соображениями или, что то же самое, желанием сделать этот список максимально "дружественным для пользователя".

ОДУ ВТОРОГО ПОРЯДКА:

$$
\begin{aligned}
& \zeta^{\prime \prime}=\left(\zeta^{\prime}\right)^{2} \zeta^{-1} f\left(\left(\zeta^{\prime}\right)^{p} \zeta^{q-p}\right), \quad 0<p \leqslant q ; \\
& \zeta^{\prime \prime}=\left(\zeta^{\prime}\right)^{2} f(\zeta), \quad p=0, \quad q=1 ; \\
& \zeta^{\prime \prime}=c(q, 1) \zeta^{\prime} \zeta^{q}+c(2 q+1,0) \zeta^{2 q+1}, \quad p=-1 \text {. }
\end{aligned}
$$

ОДУ ТРЕТЬЕГО ПОРЯДКА:

$$
\begin{aligned}
\zeta^{\prime \prime \prime} & =\zeta^{\prime \prime} \zeta^{\prime} \zeta^{-1} f\left(\left(\zeta^{\prime \prime}\right)^{p} \zeta^{2 q-p},\left(\zeta^{\prime}\right)^{p} \zeta^{q-p}\right), \quad 0<p \leqslant q ; \\
\zeta^{\prime \prime \prime} & =\zeta^{\prime \prime} \zeta^{\prime} f_{1}(\zeta)+\left(\zeta^{\prime}\right)^{3} f_{2}(\zeta), \quad p=0, \quad q=1 ; \\
\zeta^{\prime \prime \prime} & =c(q, 0,1) \zeta^{\prime \prime} \zeta^{q}+c(q-1,2,0)\left(\zeta^{\prime}\right)^{2}+c(2 q, 1,0) \zeta^{\prime} \zeta^{2 q}+ \\
& \quad+c(3 q+1,0,0) \zeta^{3 q+1}, \quad p=-1 .
\end{aligned}
$$

ОДУ ЧЕТВЕРТОГО ПОРЯДКА:

$$
\begin{aligned}
\zeta^{\prime \prime \prime \prime}= & \zeta^{\prime \prime \prime} \zeta^{\prime} \zeta^{-1} f\left(\left(\zeta^{\prime \prime \prime}\right)^{p} \zeta^{3 q-p},\left(\zeta^{\prime \prime}\right)^{p} \zeta^{2 q-p},\left(\zeta^{\prime}\right)^{p} \zeta^{q-p}\right), \\
\zeta^{\prime \prime \prime \prime}= & \zeta^{\prime \prime \prime} \zeta^{\prime} f_{1}(\zeta)+\left(\zeta^{\prime \prime}\right)^{3} f_{2}(\zeta)+\zeta^{\prime} \zeta^{2} f_{3}(\zeta)+\zeta^{4} f_{4}(\zeta), \quad p=0, \quad q=1 ; \\
\zeta^{\prime \prime \prime \prime}= & c(q, 0,0,1) \zeta^{\prime \prime \prime} \zeta^{q}+c(q-1,1,1,0) \zeta^{\prime \prime} \zeta^{\prime} \zeta^{q-1}+c(2 q, 0,1,0) \zeta^{\prime \prime} \zeta^{2 q}+ \\
& +c(q-3,3,0,0)\left(\zeta^{\prime}\right)^{3} \zeta^{q-3}+c(2 q-2,2,0,0)\left(\zeta^{\prime}\right)^{2} \zeta^{2 q-2}+ \\
& +c(3 q-1,1,0,0) \zeta^{\prime} \zeta^{3 q-1}+c(4 q, 0,0,0) \zeta^{4 q}, \quad p=-1 .
\end{aligned}
$$

Применение нашего подхода к некоторым частным видам ОДУ, которые принадлежат к классам, рассматриваемым в данной статье, уже обсуждалось в [5] и, кроме того, в работах [9], [10], где рассматривались два “классических" нелинейных ОДУ второго 
порядка. В заключение этого раздела рассмотрим еще одно "классическое" ОДУ, которое недавно было довольно полно изучено (см., например, [13]). Первоначально это уравнение было введено Чези [11] как пример ОДУ, решения которого характеризуются естественной гранищей, если рассматривать их как аналитические функции независимых переменных. Ясно, что это уравнение Чези третьего порядка,

$$
\zeta^{\prime \prime \prime}=2 \zeta^{\prime \prime} \zeta-3\left(\zeta^{\prime}\right)^{2}
$$

является просто частным случаем уравнения (3.6), где

$$
q=1, \quad c(1,0,1)=2, \quad c(0,2,0)=-3, \quad c(2,1,0)=c(4,0,0)=0,
$$

а его деформированная версия имеет вид (см. (1.5))

$$
\dddot{z}-6 i \omega \ddot{z}-11 \omega^{2} \dot{z}+6 i \omega^{3} z=2 \ddot{z} z-3 \dot{z}^{2}-\omega^{2} z^{2}
$$

\section{4. ТОЧНЫЕ ОЦЕНКИ ДЛЯ УРАВНЕНИЯ ЧЕЗИ}

Общее решение уравнения (3.12), как и решение деформированного ОДУ (3.14), известно, но только в неявной форме, включающей гипергеометрическую функцию [11], [13]. Из этой неявной формы не так легко получить информацию о свойствах периодичности решений уравнения (3.14). С другой стороны, легко доказать следуюшую теорему.

ТЕОРема. Пусть $\Sigma$ - полуалгебраическое множество, которое на пространстве начальных значений $\left(\zeta(0), \zeta^{\prime}(0), \zeta^{\prime \prime}(0)\right)$ определяется неравенствами

$$
\begin{gathered}
|\zeta(0)|<\frac{\omega}{16} \\
3\left|\zeta^{\prime}(0)\right|^{2}<2\left|\zeta^{\prime \prime}(0) \zeta(0)\right| \\
\left|\zeta^{\prime \prime}(0)\right|^{2}+8\left|\zeta^{\prime \prime}(0) \zeta^{\prime}(0) \zeta(0)\right|+12\left|\zeta^{\prime}(0) \zeta(0)\right|<8\left|\zeta^{\prime \prime}(0) \zeta^{3}(0)\right| .
\end{gathered}
$$

Ясно, что $\Sigma$ - это непустое открытое множество, и если начальные значения (недеформированного) ОДУ Чези (3.12) принадлежат множеству $\Sigma$, то решение с әтими начальными значениями является голоморфным в замкнутом круге $D$ paдuyca

$$
\tau_{D}>\frac{2}{\omega}
$$

с иентром в точке $\tau=0$ комплексной $\tau$-плоскости.

Как было показано в разделе 2, этого достаточно, для того чтобы гарантировать, что соответствуюшие решения деформированного ОДУ Чези (3.14) являются вполне периодическими, $z(t+T)=z(t)$, и имеют период $T=2 \pi / \omega$ (заметим, что переход от 
ОДУ Чези (3.12) к его деформированной версии (3.14) достигается применением нашего подхода (2.1) при $p=-1$ и $q=1)$. Теперь, выполняя тривиальные, но скучные алгебраические вычисления, с помощью (2.5) или (2.2), надо преобразовать условия (4.1) в соответствующие условия на начальные значения для деформированного ОДУ Чези (3.14). Также из рассуждений, с помощью которых в доказательстве теоремы (см. ниже) показано, что начальные значения для недеформированных ОДУ Чези (3.12), удовлетворяющие условиям (4.1), занимают открытое множество, немедленно следует, что то же самое верно и для соответствуюших начальных значений для деформированного ОДУ Чези (3.14).

ДокАЗАТЕЛЬСтво ТЕоремЫ. Запишем уравнение Чези третьего порядка (3.12) как систему трех ОДУ первого порядка, положив

$$
\begin{aligned}
& w_{0}(\tau)=b \alpha^{-1}[\zeta(\tau)-\zeta(0)], \\
& w_{1}(\tau)=b \beta^{-1}\left[\zeta^{\prime}(\tau)-\zeta^{\prime}(0)\right], \\
& w_{2}(\tau)=b \gamma^{-1}\left[\zeta^{\prime \prime}(\tau)-\zeta^{\prime \prime}(0)\right],
\end{aligned}
$$

где предполагается, что все константы $\alpha, \beta, \gamma, b$ nоложительнье. С другой стороны, мы оставляем за собой право выбирать их значения из соображений удобства (см. ниже). Таким образом, уравнение Чези третьего порядка (3.12) записывается в следующей стандартной форме:

$$
w_{j}^{\prime}(\tau)=F_{j}, \quad j=0,1,2
$$

где

$$
\begin{gathered}
F_{0}=b \alpha^{-1}\left[\zeta^{\prime}(0)+\beta b^{-1} w_{1}\right], \\
F_{1}=b \beta^{-1}\left[\zeta^{\prime \prime}(0)+\gamma b^{-1} w_{2}\right], \\
F_{2}=b \gamma^{-1}\left\{2\left[\zeta^{\prime \prime}(0)+\gamma b^{-1} w_{2}\right]\left[\zeta(0)+\alpha b^{-1} w_{0}\right]-3\left[\zeta^{\prime}(0)+\beta b^{-1} w_{1}\right]^{2}\right\},
\end{gathered}
$$

и начальные значения для этого уравнения имеют стандартный вид (см. (4.3)):

$$
w_{j}(0)=0, \quad j=0,1,2 \text {. }
$$

Из стандартных результатов (см. [12]) мы получаем следующую нижнюю грань для радиуса $\tau_{D}$ кругового диска $D$ с центром в начале координат $\tau=0$ комплексной $\tau$-плоскости, внутри которого решения $w_{j}(\tau)$ уравнений (4.4) с условиями $(4.5)$, а также (см. $(4.3))$ решение $\zeta(\tau)$ уравнения Чези (3.12) голоморфнь:

$$
\tau_{D}>\frac{b}{4 M(b)}
$$

(Эта формула с точностью до тривиальных изменений в обозначениях совпадает с последним уравнением раздела 12.21 в [12] при условии, что $m=3$ и $a=\infty$. Первое из 
этих соотношений следует из того, что система (4.4) включает три ОДУ, а второе - из того, что эта система ОДУ имеет автономный характер.) Две положительные величины $b$ и $M(b)$ в неравенстве (4.7) определяются следуюшим образом. Величина $b$ выбирается так, чтобы все три величины $F_{j}$ (см. ниже (4.9)) были голоморфнымии, если выполняются следующие три неравенства:

$$
\left|w_{j}\right| \leqslant b, \quad j=0,1,2
$$

В нашем случае это предположение не накладывает никаких ограничений на величину $b$ (поскольку три функции $F_{j}$ являются полиномиальными относительно трех переменных $w_{k}$ ). Таким образом, мы оставляем за собой право давать переменной $b$ любое произвольное положительное значение. Величина $M(b)$ является верхней гранью модулей трех (комплексных) величин $F_{j}$ (см. $\left.(4.5)\right)$, если $w_{j}$ удовлетворяют условиям (4.8),

$$
M(b)=\operatorname{Max}_{j=1,2,3 ;\left|w_{k}\right|<b}\left|F_{j}\right|
$$

конечно, неравенство (4.7) заведомо выполняется, если мы переоценим $M(b)$, что мы сейчас и сделаем.

Действительно, из (4.3) и (4.5) следует, что

$$
\begin{aligned}
M(b)< & b \operatorname{Max}\left[\alpha^{-1}\left(\left|\zeta^{\prime}(0)\right|+\beta\right), \beta^{-1}\left(\left|\zeta^{\prime \prime}(0)\right|+\gamma\right),\right. \\
& \left.\gamma^{-1}\left[2\left(\left|\zeta^{\prime \prime}(0)\right|+\gamma\right)(|\zeta(0)|+\alpha)+3\left(\left|\zeta^{\prime}(0)\right|+\beta\right)^{2}\right]\right],
\end{aligned}
$$

где максимум берется по трем значениям аргумента функции Мах. Мы по-прежнему сохраняем возможность давать произвольные значения четырем величинам $\alpha, \beta, \gamma, b$, но они все по-прежнему должны быть положительными, что, конечно, является существенным условием для получения неравенства (4.10).

Теперь для удобства положим

$$
\begin{aligned}
& \beta=\left[2\left|\zeta^{\prime \prime}(0) \zeta(0)\right|+\left|\zeta^{\prime}(0)\right|^{2}\right]^{1 / 2}-2\left|\zeta^{\prime}(0)\right|, \\
& \alpha=\frac{\left|\zeta^{\prime}(0)\right|+\beta}{2|\zeta(0)|}, \\
& \gamma=2 \beta|\zeta(0)|-\left|\zeta^{\prime \prime}(0)\right| .
\end{aligned}
$$

Заметим, что из условия (4.1в) следует, что $\beta$ (см. (4.11a)) является положительной величиной, тогда положительность величины $\alpha$ автоматически следует из предположения (4.11б), а положительность величины $\gamma$ (см. (4.11в)) следует из (4.1a) и (4.11a). С другой стороны, из условий (4.11) видно, что все три аргумента функции Мах в правой части неравенства (4.10) равны друг другу. Таким образом, из этого неравенства получаем, что $M(b)<b \omega / 2$, что позволяет доказать с помощью (4.7) неравенство (4.2). Осталось показать, что три неравенства (4.1) взаимно согласованны и что требование, 
чтобы начальные значения $\zeta(0), \zeta^{\prime}(0), \zeta^{\prime \prime}(0)$ удовлетворяли этим неравенствам, действительно ограничивает эти значения некоторой областью ненулевой меры. Для этого положим

$$
\begin{aligned}
|\zeta(0)| & =\frac{\omega}{16}(1-\varepsilon), \\
\left|\zeta^{\prime}(0)\right| & =\left(\frac{\omega}{8}\right)^{2} \eta \delta, \\
\left|\zeta^{\prime \prime}(0)\right| & =3\left(\frac{\omega}{8}\right)^{3} \eta^{2}(1+\eta) \frac{\delta^{2}}{1-\varepsilon}
\end{aligned}
$$

где $\varepsilon, \eta$ и $\delta$ - малье положительные параметры,

$$
0<\varepsilon \ll 1, \quad 0<\eta \ll 1, \quad 0<\delta \ll 1 .
$$

Легко видеть, что три неравенства (4.1) принимают вид соответственно

$$
\begin{gathered}
\varepsilon>0 \\
\eta>0 \\
(1-\varepsilon)^{4}>\delta(1+\eta)\left[4(1-\varepsilon)^{2}+3 \delta \eta(1+\eta)\right] .
\end{gathered}
$$

Следовательно, очевидно, что они имеют место при условии, что выполняются неравенства (4.13); ясно, что условия (4.12) вместе с (4.13) ограничивают (вообще говоря, комплексные) начальные значения $\zeta(0), \zeta^{\prime}(0), \zeta^{\prime \prime}(0)$ некоторой областью ненулевой меры. Что и требовалось доказать.

\section{ПРИЛОЖЕНИЕ}

Здесь мы приводим некоторые вычисления, которые в основном тексте отвлекали бы внимание от нашего изложения.

Сначала докажем соотношения (1.10) и (1.11). Подставляя (1.11a) в (1.1a), получим

$$
\begin{aligned}
& G\left(c(r)_{n} \vartheta^{r-n}+\varepsilon \varphi_{n}, \ldots, c(r)_{m} \vartheta^{r-m}+\varepsilon \varphi_{m}, \ldots, c \vartheta^{r}+\varepsilon \varphi\right) \equiv \\
& \quad \equiv G\left(c(r)_{n} \vartheta^{r-n}\left\{1+\varepsilon \varphi_{n} \vartheta^{n-r}\left[c(r)_{n}\right]^{-1}\right\}, \ldots, c \vartheta^{r}\left\{1+\varepsilon \varphi \vartheta^{-r} c^{-1}\right\}\right)=0
\end{aligned}
$$

где введены обозначения

$$
\begin{gathered}
\vartheta \equiv \tau-\tau_{b}, \\
\varphi_{m} \equiv \varphi_{m}(\tau) \equiv \frac{\partial^{m} \varphi(\tau)}{\partial \tau}, \quad m=1,2, \ldots, n,
\end{gathered}
$$

и использованы обозначения (1.10в). 
Теперь применим $r$-сортирующее свойство (1.1б) и из (П.1а) получим соотношение

$$
\begin{aligned}
& G\left(c(r)_{n}\left\{1+\varepsilon \varphi_{n} \vartheta^{n-r}\left[c(r)_{n}\right]^{-1}\right\}, \ldots, c\left\{1+\varepsilon \varphi c^{-1}\right\}\right) \equiv \\
& \quad \equiv G\left(c(r)_{n}+\varepsilon \vartheta^{n-r} \varphi_{n}, \ldots, c(r)_{m}+\varepsilon \vartheta^{m-r} \varphi_{m}, \ldots, c+\varepsilon \varphi\right)=0
\end{aligned}
$$

При $\varepsilon=0$ из этой формулы следует, что (1.10) действительно дает решение уравнения (1.1). При малых $\varepsilon \neq 0$ с помощью (1.10б) из члена первого порядка по $\varepsilon$ в разложении выражения в левой части уравнения (П.2) получаем

$$
\sum_{m=0}^{n} g_{m} \vartheta^{m} \varphi_{m}=0
$$

где использованы обозначения (1.11г). Ясно, что это ОДУ допускает решение (1.11б), удовлетворяющее условию (1.11в).

Теперь рассмотрим соотношения, из которых можно получить коэффициенты $a_{m l} \mathrm{c}$ помошью рекуррентных вычислений. Начнем с определения (2.2д), которое запишем в виде

$$
\zeta_{m}=e^{i(p-m q) \omega t} \sum_{l=0}^{m}\left[a_{m, l}(r)(i q \omega)^{l} z_{m-l}\right], \quad m=0,1,2, \ldots,
$$

где мы полагаем

$$
a_{m, 0}=1, \quad m=0,1,2, \ldots
$$

Заметим, что везде в этом приложении для удобства обозначений мы разделяем запятой два нижних индекса в коэффициентах $a_{m, l}$.

Теперь мы дифференцируем соотношения (П.4) по $t$ и, используя (2.1a), получаем

$$
\begin{aligned}
\zeta_{m+1}= & e^{i[p-(m+1) q] \omega t}\left\{i(p-m q) \sum_{l=0}^{m}\left[a_{m, l}(r)(i q \omega)^{l} z_{m-l}\right]+\right. \\
& \left.+\sum_{l=0}^{m}\left[a_{m, l}(r)(i q \omega)^{l} z_{m+1-l}\right]\right\}, \quad m=0,1,2, \ldots
\end{aligned}
$$

Сравнивая это уравнение с выражением (П.4) (где $m$ заменяется на $m+1)$, получаем

$$
\begin{aligned}
& \sum_{l=0}^{m+1}\left[a_{m+1, l}(r)(i q \omega)^{l} z_{m+1-l}\right]=\sum_{l=0}^{m}\left[a_{m, l}(r)(i q \omega)^{l} z_{m+1-l}\right]+ \\
& \quad+i(p-m q) \sum_{l=0}^{m}\left[a_{m, l}(r)(i q \omega)^{l} z_{m-l}\right], \\
& \sum_{l=0}^{m+1}\left[a_{m+1, l}(r)(i q \omega)^{l} z_{m+1-l}\right]=\sum_{l=0}^{m}\left[a_{m, l}(r)(i q \omega)^{l} z_{m+1-l}\right]+ \\
& \quad+i(p-m q) \sum_{l=1}^{m+1}\left[a_{m, l-1}(r)(i q \omega)^{l-1} z_{m+1-l}\right] .
\end{aligned}
$$


Уравнение (П.7б) получается из (П.7а), если во второй сумме в правой части заменить индекс суммирования $l$ на $l-1$.

Теперь ясно, что уравнение (П.7б) не противоречит условию (П.5) (что соответствует его члену с $l=0)$. С помошью (1.1г) из него можно получить рекуррентное соотношение

$$
a_{m+1, l}=a_{m, l}+(r-m) a_{m, l-1}, \quad l=1,2, \ldots, m,
$$

вместе с "граничным условием"

$$
a_{m+1, m+1}=(r-m) a_{m, m},
$$

из которого с учетом (П.5) немедленно следует, что

$$
a_{m, m}=(r)_{m} .
$$

Соотношения (П.8) вместе с граничными условиями (П.5) и (П.10) определяют коэффициенты $a_{m, l}$ и действительно позволяют получать эти коэффициенты шаг за шагом с помощью рекуррентных вычислений.

\section{Список литературы}

[1] F. Calogero. J. Math. Phys. 1997. V. 38. P. 5711-5719.

[2] F. Calogero, J.-P. Françoise. Inverse Problems. 2001. V. 17. P. 1-8.

[3] F. Calogero. Classical Many-Body Problems Amenable to Exact Treatments. Lect. Notes in Phys. Monograph. V. 66. Berlin: Springer, 2001.

[4] F. Calogero. Differential equations featuring many periodic solutions. In: Geometry and Integrability. London Math. Soc. Lecture Notes. V. 295. Eds. L. Mason, Y. Nutku. Cambridge: Cambridge Univ. Press, 2003. P. 9-20.

[5] F. Calogero, J.-P. Françoise. J. Nonlinear Math. Phys. 2002. V. 9. P. 99-125.

[6] F. Calogero. Phys. Lett. A. 2002. V. 293. P. 146-150.

[7] F. Calogero, M. Sommacal. J. Nonlinear Math. Phys. 2002. V. 9. P. 483-516.

[8] F. Calogero, J.-P. Françoise, M. Sommacal. Periodic solutions of a many-rotator problem in the plane. II. Analysis of various motions. J. Nonlinear Math. Phys. (in press).

[9] F. Calogero. J. Phys. A. 2002. V. 32. P. 1-8.

[10] F. Calogero. J. Phys. A. 2002. V. 35. P. 4249-4256.

[11] J. Chazy. Acta Math. 1911. V. 34. P. 317-385.

[12] Э. Л. Айнс. Обыкновенные дифференциальные уравнения. Харьков: ДНТВУ, 1939.

[13] N. Joshi, M. D. Kruskal. A local asymptotic method of seeing the natural barrier of the solutions of the Chazy equation. In: Applications of Analytic and Geometric Methods to Nonlinear Differential Equations. Proc. of the NATO Adv. Res. Workshop (Exeter, UK, July 14-19, 1992). NATO ASI Ser., Ser. C, Math. Phys. Sci. V. 413. Ed. P. A. Clarkson. Dordrecht: Kluwer, 1993. P. 331-339. 